Relations industrielles

Industrial Relations

Sociologie des outils de gestion : introduction à l'analyse de

l'instrumentation de gestion, Par Chiapello, Eve et Patrick

Gilbert. Paris : Éditions La Découverte, 2013, 294 pages. ISBN

978-2-7071-5145-2

\title{
Yves Hallée
}

Volume 70, numéro 2, printemps 2015

URI : https://id.erudit.org/iderudit/1031493ar

DOI : https://doi.org/10.7202/1031493ar

Aller au sommaire du numéro

Éditeur(s)

Département des relations industrielles de l’Université Laval

\section{ISSN}

0034-379X (imprimé)

1703-8138 (numérique)

Découvrir la revue

Citer ce compte rendu

Hallée, Y. (2015). Compte rendu de [Sociologie des outils de gestion:

introduction à l'analyse de l'instrumentation de gestion, Par Chiapello, Eve et

Patrick Gilbert. Paris : Éditions La Découverte, 2013, 294 pages. ISBN

978-2-7071-5145-2]. Relations industrielles / Industrial Relations, 70(2), 386-387.

https://doi.org/10.7202/1031493ar

Tous droits réservés @ Département des relations industrielles de l’Université Laval, 2015
Ce document est protégé par la loi sur le droit d'auteur. L'utilisation des services d'Érudit (y compris la reproduction) est assujettie à sa politique d'utilisation que vous pouvez consulter en ligne.

https://apropos.erudit.org/fr/usagers/politique-dutilisation/ 
seuls à n'avoir aucun intérêt pour les préoccupations du monde syndical et ouvrier. Les dommages seront notables, et, pourrait-on ajouter, ils le sont encore aujourd'hui.

L'ouvrage de David Frank s'avère donc méritoire. Bien que son objet lui-même soit I'histoire de la FTTNB, nous devons inscrire ce livre dans l'histoire - dont plusieurs chapitres restent à écrire - du syndicalisme au Nouveau-Brunswick et, plus généralement encore, de I'histoire de la gauche dans la province. II s'agit d'un effort essentiel qui déborde largement (ce sera au lecteur de juger s'il s'agit là d'une qualité ou d'un défaut de l'ouvrage) les cadres initiaux de son objet : en effet, au lieu d'une histoire stricte de la FTTNB, Frank nous offre plutôt une histoire partielle, ouvrière et syndicale, de I'histoire du Nouveau-Brunswick au XX' ${ }^{\top}$ siècle.

\section{Julien Massicotte}

Professeur

Campus d'Edmundston

Université de Moncton

\section{Sociologie des outils de gestion : introduction à l'analyse de I'instrumentation de gestion.}

Par Chiapello, Eve et Patrick Gilbert. Paris : Éditions La Découverte, 2013, 294 pages. ISBN 978-2-7071-5145-2.

Cet ouvrage de Chiapello et Gilbert propose une analyse sociale des outils de gestion, ce qui permet de sortir de « l'enfermement pratico-utilitaire » dans lequel sont souvent confinées plusieurs études (p. 13). La technique est alors considérée " comme un fait social total » (p. 13), une perspective redevable à I'analyse sociologique et anthropologique, ce qui rend possible le questionnement sur « les représentations sociales, les systèmes de valeurs, les règles du jeu, les rapports de force qui éclairent les actes de gestion, voire les expliquent » (p. 13). Les auteurs reconnaissent " comme outil de gestion un ensemble délimité d'objets organisationnels dotés de traits caractéristiques qui s'offrent à une triple description : fonctionnelle, structurelle et processuelle » (p. 32).
Leur revue des diverses conceptualisations théoriques débute par les approches traditionnelles qui découlent de la théorie des organisations. Par la suite, sont abordées les approches critiques, institutionnalistes et interactionnelles. Finalement, en troisième partie, nous avons une série d'études de cas qui illustrent ces développements théoriques.

Les courants associés aux perspectives traditionnelles fondent leur analyse sur « le fonctionnement, la structure et le développement des organisations afin d'en améliorer la performance » (p. 37). Le propos de Chapiello et Gilbert s'amorce avec le management classique (Taylor et Fayol), pour ensuite aborder les écoles rationnelles (Simon et la rationalité limitée), et en arriver à l'approche contingente, que les auteurs associent aux théories rationnelles. Un passage par l'école des relations humaines et le comportement organisationnel - évoqué comme étant du déni de la technique, mais associé aux théories normatives - est ensuite réalisé. Les auteurs concluent à la prégnance des croyances rationnelles, lesquelles conforteraient le gestionnaire quant à la maîtrise " des réalités mouvantes » (p. 48) que permettrait l'instrumentation de gestion. Les théories des organisations étant jugées peu fécondes pour éclairer les questions centrales, Chiapello et Gilbert les délaissent pour plutôt aborder I'approche sociotechnique (Trist et Emery) - laquelle évoque l'optimisation conjointe de la technique et du social - , de même que l'approche comportementale (Cyert et March) où est mis en exergue l'importance des routines organisationnelles, c'est-à-dire les procédures systématiques permettant la régulation de " l'ensemble de coalitions aux intérêts divergents [...] se livrant à des jeux politiques et des relations de pouvoir » (p. 52).

En deuxième partie, les auteurs discutent des perspectives critiques à laquelle est associée la thèse de la domination et de l'exploitation. Dans ce cadre, l'instrumentation 
est alors perçue comme ayant pour fonction de légitimer les hiérarchies sociales. Analyse inspirée des travaux de Foucault, cette thèse présente les outils comme des instruments techniques de discipline et de gouvernance. Ils sont appréhendés en tant que " vecteur de déshumanisation du travail et d'aliénation des sujets » (p. 89). Signalons que selon cette perspective, l'instrumentation évacue une part notable du réel, tout en faisant fi de la complexité et de la subjectivité des acteurs.

Les auteurs traitent ensuite du rôle des institutions dans la formation, le développement et les effets des outils de gestion. Tout d'abord, Chiapello et Gilbert analysent les conséquences de l'approche néo-institutionnaliste, notamment le concept de champ organisationnel qui amène les organisations à adopter des pratiques similaires parce qu'elles " cherchent à apparaître légitimes en se conformant aux modèles dominants de ce qui est socialement accepté ou valorisé » (p. 99). Pour les conventionnalistes, I'outil est un investissement de forme dans la mesure où des conventions permettent de réduire l'incertitude et guident les jugements à partir d'épreuves qui y sont incorporés. Cette section se termine avec la théorie de la structuration de Giddens où « les systèmes sociaux ne peuvent être compris indépendamment des structures sociales qui les façonnent » (p. 131). Deux thèses sont analysées: la première stipule que les outils de gestion possèdent un potentiel de structuration s'exprimant lors de leur utilisation alors que la seconde s'appuie sur l'idée que les structures sont virtuelles ce qui fait « que le rôle des outils de gestion dans le processus de structuration n'est qu'indirect » (p. 131).

Quant aux approches interactionnelles, les thèses abordées écartent le déterminisme externe pour mettre l'emphase sur I'autonomie des acteurs. La thèse de la théorie de l'acteur réseau fait référence à la " coconstruction», selon laquelle "les objets et les utilisateurs sont unis par un faisceau de liens; [ainsi,] pour qu'un outil de gestion réussisse à s'implanter, des adaptations multilatérales et une recherche de compromis impliquant les humains et les non humains sont nécessaires » (p. 147). Une autre thèse, celle se référant à " l'outil comme être de langage » nous apprend notamment qu'il " n'y a pas de transformation technologique qui ne soit accompagnée de modification du langage » (p. 162). Enfin, la thèse de la théorie de l'acteur stratégique de Crozier et Friedberg analyse les outils de gestion dans une perspective de soumission au jeu des acteurs. Les spécialistes (informaticiens, ingénieurs de méthode, etc.) tendent à concevoir le futur organisationnel sur la base de postulats servant leurs intérêts d'acteurs. On réfute l'idée que l'autonomie des acteurs disparaît sous la pression des outils de gestion (norme ISO, juste à temps, qualité totale, etc.); par conséquent, le changement ne serait effectif que si " les acteurs trouvent un sens à I'action qu'ils entreprennent » (p. 173).

Le volume de Chiapello et Gilbert se caractérise par un souci pédagogique. Chacune des thèses défendues est agrémentée de tableaux portant sur des publications représentatives documentées ainsi que de fiches synthèses sur les axiomes, les concepts-clés, les règles de la méthode, les figures de style, les auteurs et les textesclés. Aussi, le recours à des études de cas apporte un éclairage empirique nécessaire aux différentes perspectives théoriques. II s'agit d'un ouvrage de synthèse généreux par l'ampleur de sa couverture théorique. Petit bémol cependant, certaines thèses auraient gagné à être davantage explicitées afin de permettre une meilleure compréhension. Nonobstant cette remarque, nous saluons cette contribution de Chiapello et Gilbert sur les outils de gestion, lesquels sont, hélas, trop souvent abordés dans une perspective strictement utilitaire.

\section{Yves Hallée}

Professeur agrégé

Département des relations industrielles

Université Laval 